\section{Correspondence on 'Ultrasound shows rapid reduction of crystal depositions during a treat- to-target approach in gout patients: 12-month results from the NOR-Gout study'}

With great interest, we read the article by Hammer et al reporting a treat-to-target (T2T) approach with uratelowering treatment (ULT) resulted in significant reductions of crystal depositions via ultrasonic detection. ${ }^{1}$ We agree with the authors that the use of new semiquantitative scoring system may increase the sensitivity to minor changes in lesions in contrast to a binary scoring system. We would like, however, to highlight some key points.

First and foremost, there is no control group in this study. Although patients with gout have rapid reduction of crystal depositions by a T2T approach, we cannot tell how much of this improvement is due to the effect of the treatment itself. Moreover, whether the operators and the participants are blind or not is unknown in the article. A double-blind, controlled study is essential for giving information on effectiveness. Next, there are medicines left out, and a subgroup analysis for different treatment is required. Allopurinol and febuxostat are the medication with ULT in the experiment. However, we caution that benzbromarone is another common medication when patients have intolerance for allopurinol and febuxostat. Previous researchers have taken benzbromarone in consideration during ULT research. ${ }^{2}$ Also, the proportions and a subgroup analysis of patients using different medicines (allopurinol and febuxostat) are recommended for verifying effectiveness. Last but not the least, according to European League Against Rheumatism (EULAR), a lower target (under 5 $\mathrm{mg} / \mathrm{dL}$ or $300 \mu \mathrm{mol} / \mathrm{L}$ ) might be given if patients have severe gout with tophi, chronic arthropathy or very frequent attacks. In normal situation, the target of serum urate level is set up by $6 \mathrm{mg} / \mathrm{dL}$. We note that the proportion of the patients whose target is under $5 \mathrm{mg} / \mathrm{dL}$ is unsaid, and that the results should be presented separately. ${ }^{3}$

There may be some information bias in the research. On the one hand, the locations for elementary lesions in Table 1 may be underestimated. Locations such as kidneys, tibialis posterior tendons, ${ }^{2}$ peroneus, metacarpophalangeal joints (MCP)1, MCP3-5 and metatarsophalangeal joints (MTP)2-5 are not shown in your results ${ }^{2}$ which may lead to inaccurate detection and wrong conclusions. On the other hand, erosions, one of the elementary lesions according to Outcome Measures in Rheumatology (OMERACT), have been missed out in Table 2. Since the sum scores of double contours, tophi and aggregates cannot represent all of the elementary lesions of patients with gout, we propose that erosions should be taken into account as an outcome during 12 months follow-up as in previous studies. ${ }^{245}$ Another information bias is the unknowingness of the periods of recruitment, exposure and data collection. Besides, the medical institution where the experiment took place is yet to be clarified. We are looking forward to understanding further details.

Apart from the above, we are sincerely concerned about some selection bias and residual confounders in the situation. First, both patients being naïve to ULT or with previous or present ULT treatment were included in this study. However, the patients who have undergone ULT, or even by a T2T approach, before the enrolment may be a confounder. We suggest that a subgroup analysis for naïve, previous and present users is needed. Second, the rules of study patient enrolment may be inadequate. The inclusion and exclusion criteria in previous studies like age $\geq 18$ years, $^{26}$ not to have recent corticosteroid ${ }^{6}$ or glucocorticoid ${ }^{2}$ injections before study entry, and no history of severe renal insufficiency, psoriasis, drug-induced gout and other secondary gout types ${ }^{6}$ are not mentioned in the article. Finally, alcohol consumption, ${ }^{26}$ smoking history, ${ }^{26}$ comorbidities $^{278}$ and body mass index ${ }^{68}$ may be some residual confounders unrevealed in the experiment. More investigation and stratification are expected to remove confounding bias.

To sum up, we are convinced that a double-blind, controlled study with different subgroups is necessary to enhance credibility. An outcome of erosions and some undetected locations should be included. Lastly, the enrolment criteria and residual confounders are requested to explication corresponding to your great work.

Po-Cheng Hung $\odot,{ }^{1}$ Da-Hung Lin $\odot,{ }^{1}$ Amy Ker $\odot,{ }^{1}$ Chieh-Chun Yang $\odot,{ }^{1}$ Hung-Wan Chien, ${ }^{2}$ James Cheng-Chung Wei $\circledast^{3,4,5}$

${ }^{1} S c h o o l$ of Medicine, Chung Shan Medical University, Taichung, Taiwan ${ }^{2}$ Department of Emergency Medicine, Chung Shan Medical University Hospital, Taichung, Taiwan

${ }^{3}$ Department of Allergy, Immunology \& Rheumatology, Chung Shan Medical University Hospital, Taichung, Taiwan

${ }^{4}$ Institute of Medicine, College of Medicine, Chung Shan Medical University, Taichung, Taiwan

${ }^{5}$ Graduate Institute of Integrated Medicine, China Medical University, Taichung, Taiwan

Correspondence to Dr James Cheng-Chung Wei, Institute of Medicine, College of Medicine, Chung Shan Medical University, Taichung City 40201, Taiwan; jccwei@gmail.com

Contributors All other authors (PCH, DHL, AK, CCY, HWC and JCW) provided their input by contributing to the conceptualisation. $\mathrm{PCH}$ and HWC contributed to the editing of the manuscript. PCH and HWC contributed equally.

Funding The authors have not declared a specific grant for this research from any funding agency in the public, commercial or not-for-profit sectors.

Competing interests None declared.

Patient and public involvement Patients and/or the public were not involved in the design, or conduct, or reporting, or dissemination plans of this research.

Patient consent for publication Not required.

Provenance and peer review Not commissioned; internally peer reviewed.

(C) Author(s) (or their employer(s)) 2020. No commercial re-use. See rights and permissions. Published by BMJ.

\section{Check for updates}

To cite Hung P-C, Lin D-H, Ker A, et al. Ann Rheum Dis Epub ahead of print: [please include Day Month Year]. doi:10.1136/annrheumdis-2020-218846

Received 14 August 2020

Accepted 16 August 2020

\section{Linked}

- http://dx.doi.org/10.1136/annrheumdis-2020-218908

Ann Rheum Dis 2020;0:1-2. doi:10.1136/annrheumdis-2020-218846

ORCID iDs

Po-Cheng Hung http://orcid.org/0000-0002-4201-6321

Da-Hung Lin http://orcid.org/0000-0002-0584-948X

Amy Ker http://orcid.org/0000-0002-7756-3878

Chieh-Chun Yang http://orcid.org/0000-0003-4500-7540

James Cheng-Chung Wei http://orcid.org/0000-0003-0310-2769

\section{REFERENCES}

1 Hammer HB, Karoliussen L, Terslev L, et al. Ultrasound shows rapid reduction of crystal depositions during a treat-to-target approach in gout patients: 12-month results from the NOR-Gout study. Ann Rheum Dis;18:annrheumdis-2020-217392. 
2 Christiansen SN, Østergaard M, Slot O, et al. Assessing the sensitivity to change of the OMERACT ultrasound structural gout lesions during urate-lowering therapy. RMD Open 2020;6:e001144.

3 Dalbeth N, Billington K, Doyle A, et al. Effects of allopurinol dose escalation on bone erosion and urate volume in gout: a dual-energy computed tomography imaging study within a randomized, controlled trial. Arthritis Rheumatol 2019;71:1739-46.

4 Cazenave T, Martire V, Reginato AM, et al. Reliability of OMERACT ultrasound elementary lesions in gout: results from a multicenter exercise. Rheumatol Int 2019;39:707-13.
5 Christiansen S, Østergaard M, Slot O, et al. FRI0229 The validity of the omeract ultrasound definitions of gout elementary lesions in the diagnosis of gout. Ann Rheum Dis 2018;77:656.

6 Naredo E, Uson J, Jiménez-Palop M, et al. Ultrasound-detected musculoskeletal urate crystal deposition: which joints and what findings should be assessed for diagnosing gout? Ann Rheum Dis 2014:73:1522-8.

7 Kuo C-F, Grainge MJ, Zhang W, et al. Global epidemiology of gout: prevalence, incidence and risk factors. Nat Rev Rheumatol 2015;11:649-62.

8 Kuo C-F. A changing landscape of gout: comorbidity matters. J Rheumatol 2018;45:454-5 